\title{
Immunomodulatory Agent CC-11006
}

National Cancer Institute

\section{Source}

National Cancer Institute. Immunomodulatory Agent CC-11006. NCI Thesaurus. Code C101370.

A proprietary, orally available, small molecule and thalidomide analog, with potential immunomodulating and antineoplastic activity. CC-11006 appears to have a similar mechanism to thalidomide and may modulate the expression of proinflammatory and regulatory cytokines. 